\title{
Parotid Sialolithiasis: A Case Report
}

\author{
Dr. Soumithran C.S ${ }^{1}$, Dr. Manu Mathew ${ }^{2}$, Dr. Arul Dev D.P ${ }^{3}$, \\ Dr. Mithilesh K.V ${ }^{4}$, Dr. Fazmiya ${ }^{5}$ \\ ${ }^{\text {I }}$ Professor \& HOD, Dept. Of OMFS, GDC Kozhikode, India) \\ ${ }^{2}$ (Junior Resident, Dept. Of OMFS, GDC Kozhikode, India) \\ ${ }^{3}$ (Junior Resident, Dept. Of OMFS, GDC Kozhikode, India) \\ ${ }^{4}$ (Junior Resident, Dept. Of OMFS, GDC Kozhikode, India) \\ ${ }^{5}$ (Junior Resident, Dept. Of OMFS, GDC Kozhikode, India)
}

\begin{abstract}
Sialolithiasis is a disease which affects the salivary glands and is characterized by the obstruction of the gland or its excretory duct due to the formation of calcified structures. The development of sialoliths is a multifactorial event. They are more often located within the ductal system of the submandibular gland $(72 \%$ 95\%) than in the ductal system of the parotid gland (4\%-28\%). The present study reports an uncommon case of sialolithiasis in a 45-year-old male patient who presented with swelling and pain in the right parotid duct and was treated by surgical removal of the stone via an intraoral approach under local anaesthesia.
\end{abstract}

Keywords: sialolithiasis, stenson's duct, salivary gland, local anaesthesia

\section{Introduction}

Sialolithiasis is a condition characterized by the obstruction of a salivary gland or its excretory duct due to the formation of calcareous concretions or sialoliths[1]. It is usually associated with swelling, pain, and infection of the affected gland resulting in salivary ectasia[2]. It is the most common disease of the major salivary glands after mumps and accounts for approximately $30 \%$ of all salivary disorders. About $0.01-1.0 \%$ of the population is said to be affected, with a higher incidence in males aged between 30 and 60 years [3]. More than $80 \%$ of salivary sialoliths occur in the submandibular duct or gland, $6-15 \%$ occur in the parotid gland and around $2 \%$ are in the sublingual and minor salivary glands[4]. It causes mechanical obstruction and swelling of the salivary glands. A further effect may be the infection of the salivary gland which may result in chronic sialadenitis.

The exact aetiology of sialolith formation remains unknown. Several hypotheses have been put forward to explain the etiology of these calculi: mechanical, inflammatory, chemical, neurogenic, infectious, etc[1]. But it is thought that more alkaline, viscous, mucus rich saliva,containing higher percentage of calcium phosphates as in submandibular salivary gland favours the formation of sialolith. In addition the long and sinuous position of Wharton's duct too contributes to stasis making the submandibular salivary system more prone to the development of sialoliths than the parotid gland. It is known that systemic diseases (gout, Sjögrens), medications (anticholinergics, antisialogogues), local trauma, head and neck radiotherapy, being elderly and renal impairment also can predispose patients to sialolith formation[4]. A combination of variety of these factors usually provokes the precipitation of the amorphous tricalcic phosphate, which, once crystallized and transformed into hydroxyapatite becomes the initial focus. From this moment on, it acts as a catalyst that attracts and supports the proliferation of new deposits of different[1]. Most salivary calculi are small and usually less than $1 \mathrm{~cm}$, but megaliths or giant calculi have been reported.

Management of sialoliths depends on the stone size, location, number of stones, and the extent of ductal obstruction[5]. Surgical management ranges from minimally invasive to open surgical techniques. Conventionally calculi in the distal section of the parotid duct near the punctum are removed through an intraoral approach. Those in the proximal duct and parenchyma pose more of a problem, particularly when sealed into the duct with strictures. The surgical option for these is parotidectomy with its attendant complications, notably injury to the facial nerve (9\%), damage to the great auricular nerve, and Frey syndome[6]. Extraoral sialolithotomy without parotidectomy was first described by Baurmarsh and Dechiara in 1991 using plain radiographs and ultrasonography[7]. Later, extra-corporeal shock wave lithotripsy (ESWL) was proposed as an alternative therapeutic approach in the treatment of salivary stones[3]. Most recently the introduction of sialoendoscopes has revolutionized the management of sialolithiasis and allowed for a more accurate diagnosis and localization of the obstruction.

\section{Case Presentation}

A 45-year-old male to the department of Oral \& Maxillofacial Surgery with complaint of recurrent swelling and pain on the patient reported right cheek since one year(Fig.1). The swelling and discomfort 
exacerbated during or before the meals and subsided by its own. He also gave a history of pus discharge through an extraoral sinus 3 months back which subsided on taking antibiotics(Fig.2). On examination, he had a swelling on the right side of the cheek of size about $3 \times 3 \mathrm{~cm}$. The swelling was warm, firm, and tender on palpation and not adhered to any deeper structure. The ductal opening was inflamed and hypertrophic and on milking there was pus discharge(Fig.3). Orthopantomography revealed a radioopaque mass of size $1.5 \times 1.5 \mathrm{~cm}$ in relation to the maxillary third molar in the stenson's duct region suggestive of sialolithiasis(Fig.5). Ultra sonographic imaging was also done which revealed bulky right parotid with enlarged intraparotid lymphnodes. Parotid duct was dilated along the entire course upto buccinator and a curvilinear echogenic mass of size $1.2 \times 0.6 \mathrm{~cm}$ was noted in the stenson's duct suggestive of sialodocholithiasis.

\section{Treatment}

Once the diagnosis was confirmed the treatment plan was to remove the sialolith under local anaesthesia. After achieving local anaesthesia,once the sialolith was located, first step of the treatment, was to achieve its immobilization by means of suture to prevent it from moving along the duct during the surgical procedure(Fig.3). An incision was placed directly over the sialolth parallel to the direction of salivary duct to expose the stone. Further dissection was done around the sialolith to separate it and was removed(Fig.4). The size was $1.2 \times 0.6 \mathrm{~cm}$. Suturing was done with $3-0$ black braided silk. Patient was then discharged with antibiotics and analgesics.

\section{Discussion}

Sialolithiasis is caused by obstruction of a salivary gland or its excretory duct by a calculus. Some sialoliths may be asymptomatic and identified incidentally during jaw imaging. But mostly they are characterized by a series of symptoms. The first one is salivary duct swelling, either without any obvious reason or at meal times. This symptom lasts for a relatively short period, no more than $2 \mathrm{hrs}$. Approximately $30 \%$ of the time, sialolithiasis presents with painless swelling[2]. But the classic symptoms are secondary to duct obstruction and include pain and swelling of the involved gland during eating when saliva production is at its maximum and salivary flow is forced against a fixed obstruction[4]. In some cases, the swelling is accompanied by an episode of salivary colic: an acute, lacerating pain that disappears after 15 or 20 minutes[2]. Subsequent gradual reduction of the swelling can occur, but as salivary flow is stimulated, painful symptoms can recur. Patients may have episodic swelling and discomfort, or may have more persistent symptoms as salivary fluid accumulates within the duct. This can lead to suppuration and sialadenitis of the involved gland[1]. There can be bad breath and foul-tasting mouth. In our case patient had recurrent episodes of swelling and pain associated with meals and pus discharge from the duct and an external sinus.

The cause of salivary stone formation remains unclear. However, there are two main theories that attempt to explain the formation of salivary stones. The first theory postulates that a local inflammatory process leads to calcification of a mucus plug. The second theory assumes that microsialoliths, produced by autophagosomes in the salivary gland, form a nidus for calcium precipitation[8]. Salivary stones are mainly composed of inorganic material, such as hydroxyapatite, carbonate apatite, whitlockite, and brushite, with smaller amounts of organic material, such as collagen, glycoproteins, lipids, and carbohydrates [8]. Crystallographic studies reveal the differences between parotid and submaxillary calculi[1]. In relation to the composition of parotid calculi ,the study conducted by Slomiany et al. mentioned a total lipidic component of $8.5 \%$ and a mineral component of $20.2 \%$. The composition and size of salivary calculi has some diagnostic implications[9]. Around $20 \%$ of submandibular gland sialoliths and $40 \%$ of parotid ones are radiolucent due to the low mineral component of the secretion, especially in the case of parotid calculi[1].

Diagnostic imaging to identify presumed salivary calculi include conventional radiography, sialography and USG. But currently, high-resolution non-contrast CT scanning is the imaging modality of choice for the evaluation of salivary stones. This is because many calcified sialoliths are not detected by conventional radiography until they are $60-70 \%$ calcified with at least $20 \%$ of submandibular and $50 \%$ of parotid stones not identifiable on intraoral and panoramic radiography[4]. Conventional radiography include both intra oral and extra oral modalities. Conventional intra-oral radiography may be useful more in detecting the submandibular sialoliths compared to the parotid due to course of the stenson's duct around the anterior portion of the masseter muscle and through the buccinator[1]. In general, only the sialoliths located in the anterior part of the duct, in front of the masseter muscle, can be visualized by means of intra-oral radiography. Extra oral radiography is also of limited use as the parotid sialoliths may be superimposed on the maxilla[1]. In sialography, a dye is injected into the duct, and it can demonstrate obstruction as a filling defect in the duct and duct stenosis[4]. It allows for the visualization of the whole duct system. However, sialography is not indicated in the case of acute infections or patients sensitive to substances containing iodine[1]. It should not be used either if a radiopaque calculus is observed in the distal portion of the duct, as this technique could move the calculus to the most proximal portion of the duct, thereby complicating its removal. Submandibular and parotid 
calculi diagnosis can be readily performed with ultrasonography, for almost all intraparenchymal stones[3]. USG identifies calculus as white echogenic structures with glandular inflammatory changes of the salivary gland[4]. In our case also the USG revealed a curvilinear echogenic mass of size $1.2 \times 0.6 \mathrm{~cm}$ in the stenson's duct suggestive of sialolith. In short, there is not a single technique for the diagnosis of salivary gland sialolithiasis and we must select the most appropriate technique according to the circumstances and pathology to be treated. We must obtain an accurate diagnosis and at the same time minimize the risk and inconvenience for the patient.

In the case of small calculi the treatment of choice should be medical instead of surgical. A conservative approach, including oral analgesia, hydration, local warm heat therapy, massage to 'milk' out the stone, sialogogues to promote ductal secretions are recommended[4]. The endoscopically-assisted technique for the retrieval of parotid stones is a viable, gland-sparing, alternative with a high success rate and few postoperative complications. Interventional sialendoscopy is successful for mobile stones and stones less than 5 $\mathrm{mm}$; fixed stones and stones larger than $5 \mathrm{~mm}$ can be managed by ESWL[10]. Open surgical management is reserved for sialoliths for which non invasive treatment failed , and depends on the stone size and location[5]. Open surgical approaches include trans oral duct incision, purely external approaches, or a combined approach. The morbidity after superficial and total parotidectomy as treatment for parotid calculi is often disproportionate to the symptoms caused. The reported risk to the facial nerve after superficial parotidectomy varies from $16 \%$ $38 \%$ for temporary weakness, and up to $9 \%$ for permanent damage[6]. The development of minimally-invasive salivary techniques have reduced the need for both parotid and submandibular sialoadenectomy for sialolithiasis. Trans oral incision directly over the stone and dissecting the stone out is an excellent minimally invasive procedure when the sialolith are in the most distal part of the duct. In our case the sialolith was placed near to the ductal opening but the larger size of stone prevented it from milking out through the duct. Hence it was removed through a transoral incision under local anaesthesia. Patient tolerated the procedure well and was discharged following the procedure.

\section{Conclusion}

In conclusion, several options are available for the management of sialolithiasis, ranging from sialoendoscopy to ductal incision, and approaches combining sialoendoscopy with a targeted open approach. These options are all gland sparing and minimally invasive strategies. . The use of one or another technique depends to a large extent on the sialolith size, location and composition. Gland excision is reserved as a last resort or in cases with multiple intra parenchymal sialoliths.

\section{Figures}

Fig. 1

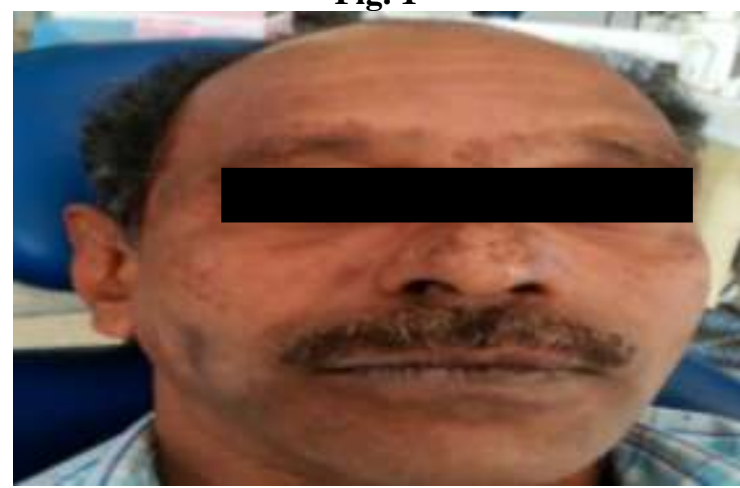

45 year old male patient with swelling on the right cheek

Fig.2

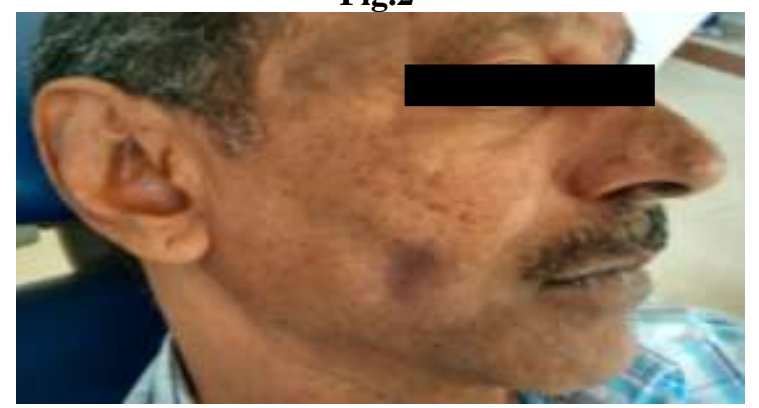

Lateral view showing a healed extra oral sinus 


\section{Fig.3}

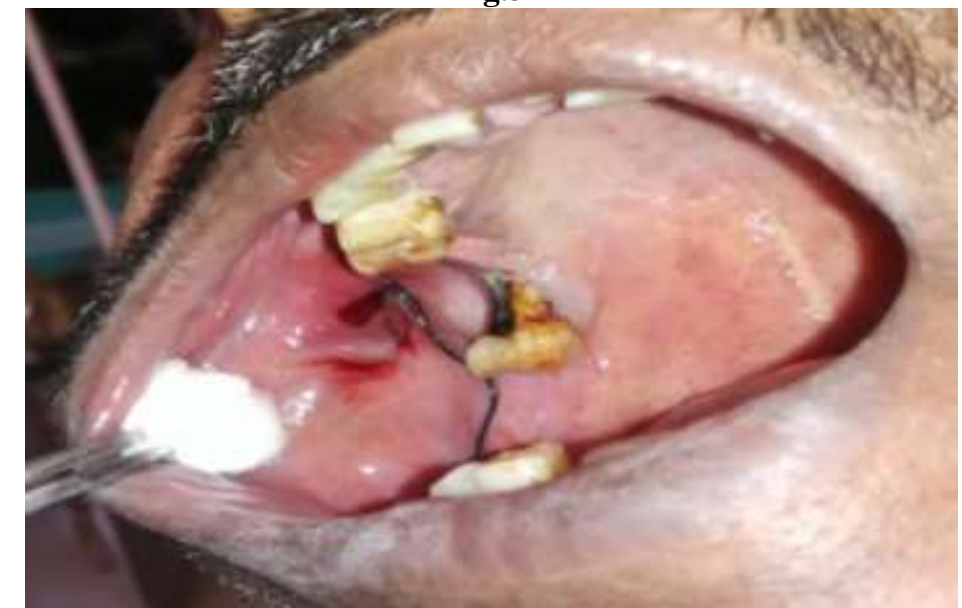

Intra operative figure showing the tie suture, also note the hypertrophic ductal opening

Fig.4

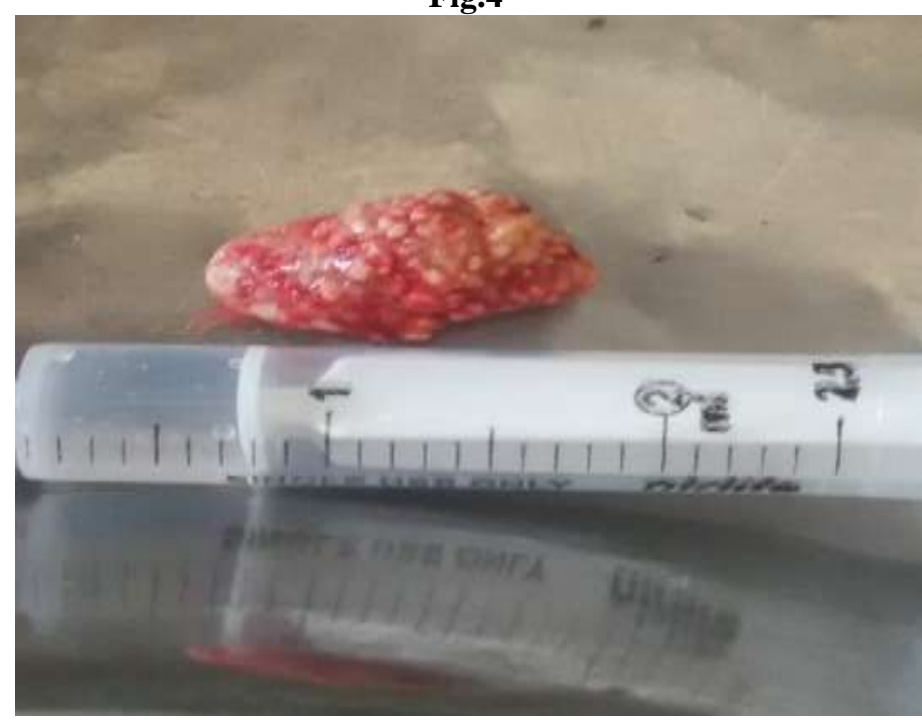

Sialolith after excision

Fig.5

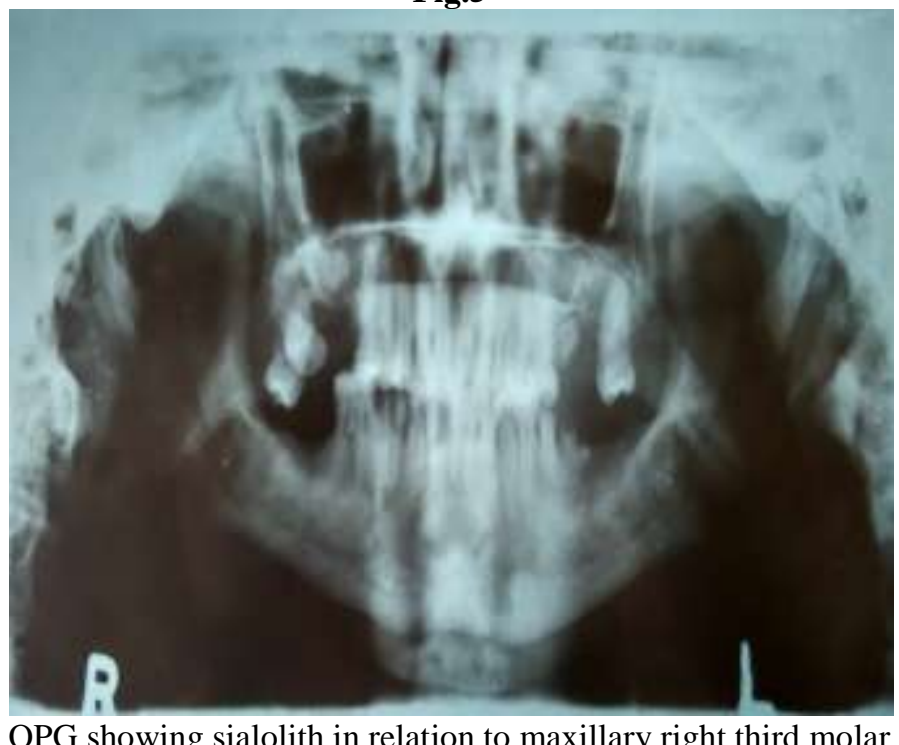

OPG showing sialolith in relation to maxillary right third molar 


\section{References}

[1]. Torres-Lagares, D., et al., Parotid sialolithiasis in Stensen's duct. Med Oral Patol Oral Cir Bucal, 2006. 11(1): p. E80-4.

[2]. Debnath, S.C. and A.K. Adhyapok, Sialolithiasis of an accessory parotid gland: an unusual case. Br J Oral Maxillofac Surg, 2015. 53(7): p. 658-9.

[3]. Andretta, M., et al., Current opinions in sialolithiasis diagnosis and treatment. Acta Otorhinolaryngol Ital, 2005. 25(3): p. 145-9.

[4]. Moghe, S., et al., Parotid sialolithiasis. BMJ Case Rep, 2012. 2012.

[5]. Sam J.Daniel, A., Open surgical management of sialolithiasis. Operative Techniques in Otolaryngology, 2015(26): p. 143-149.

[6]. Samani, M., et al., Minimally-invasive surgery in the management of symptomatic parotid stones. Br J Oral Maxillofac Surg, 2016.

[7]. Baurmash, H. and S.C. Dechiara, Extraoral parotid sialolithotomy. J Oral Maxillofac Surg, 1991. 49(2): p. 127-32.

[8]. Kraaij, S., et al., Systemic diseases and the risk of developing salivary stones: a case control study. Oral Surg Oral Med Oral Pathol Oral Radiol, 2015. 119(5): p. 539-43.

[9]. Slomiany, B.L., et al., Lipid composition of human parotid salivary gland stones. J Dent Res, 1983. 62(8): p. 866-9.

[10]. Capaccio, P., M. Gaffuri, and L. Pignataro, Sialendoscopy-assisted transfacial surgical removal of parotid stones. J Craniomaxillofac Surg, 2014. 42(8): p. 1964-9. 